\title{
ON THE EXPONENT OF CONVERGENCE OF NEGATIVELY CURVED MANIFOLDS WITHOUT GREEN'S FUNCTION
}

\author{
María V. Melián*, José M. RodríGuez*†, and Eva Tourís*
}

\begin{abstract}
In this paper we prove that for every complete $n$-dimensional Riemannian manifold without Green's function and with its sectional curvatures satisfying $K \leq-1$, the exponent of convergence is greater than or equal to $n-1$. Furthermore, we show that this inequality is sharp. This result is well known for manifolds with constant sectional curvatures $K=-1$.
\end{abstract}

2010 Mathematics Subject Classification: 53C20, 53C21, 53C22, 53C23, 30F.

Key words: Riemannian manifold, negative curvature, Green's function, first eigenvalue, exponent of convergence.

\section{Introduction}

In this paper we prove a relation between two useful concepts of Potential Theory: the existence of Green's function and the exponent of convergence, for complete Riemannian $n$-manifolds $(n \geq 2)$ with sectional curvatures $K \leq-1$. These concepts are related with other interesting topics as, for instance, the first eigenvalue of the Laplace-Beltrami operator, heat kernel, isoperimetric inequalities, conic limit set, and geodesics in the manifold (see Section 2).

We prove that for every complete $n$-dimensional Riemannian manifold without Green's function and with sectional curvatures satisfying $K \leq-1$, the exponent of convergence is greater than or equal to $n-1$. This inequality is sharp, as we show after the proof of this theorem. These results are well-known for manifolds with constant sectional curvatures $K=-1$. Note that the case of complete Riemannian $n$-manifolds with sectional curvatures $K \leq-k^{2}<0$ can be reduced to this one.

\footnotetext{
* Supported in part by four grants from Ministerio de Ciencia e Innovación (MTM 2009-07800, MTM 2008-02829-E, MTM 2013-46374-P, and MTM 2015-69323REDT), Spain.

†Supported in part by a grant from CONACYT (FOMIX-CONACyT-UAGro 249818), México.
} 
Let us recall that a Green's function in a complete Riemannian manifold $M$ is a positive fundamental solution of the Laplace-Beltrami operator $\Delta:=-\operatorname{div}$ grad on $M$. It is well-known that a complete manifold has Green's function if and only if there exists a non-constant positive superharmonic function (see, e.g., [18]). In terms of Brownian motion, a complete manifold has Green's function if and only if the Brownian motion on the manifold is transient.

Green's function is related with isoperimetric inequalities: Fernández proved in [8] that the existence of some kind of isoperimetric inequalities guarantees the existence of Green's function for Riemannian manifolds; the results in [11] imply that the linear isoperimetric inequality guarantees the existence of Green's function for negatively curved Riemannian surfaces.

Let $M$ be a complete $n$-dimensional Riemannian manifold and let us write $M=\tilde{M} / \Gamma$, where $\tilde{M}$ is a universal covering of $M$ and $\Gamma$ is a discrete group of isometries of $\tilde{M}$. The exponent of convergence $\delta(M)$ (see, e.g., [15, p. 21] for basic background) is defined as

$\delta(\Gamma):=\delta(M):=\inf \left\{t: \sum_{\gamma \in \Gamma} \exp (-t d(x, \gamma x))<\infty\right.$, for some $\left.x \in \tilde{M}\right\}$

It is easy to check that if the series converges for some $x \in \tilde{M}$, then it converges for all $x \in \tilde{M}$.

The exponent of convergence plays an important role in the study of geodesics in negatively curved manifolds. In particular, the set of bounded geodesics has Hausdorff dimension equal to the exponent of convergence (see [4], [9], and [17]). The exponent of convergence also plays an important role in the study of escaping geodesics in negatively curved surfaces (see [10] and [14]).

Besides, in [17] it was proved that the exponent of convergence of a complete negatively curved manifold is equal to the Hausdorff dimension of its conic limit set (see the definition in [20]); it was previously proved in the case of constant curvature in [16] for surfaces of finite area, and in full generality in $[\mathbf{9}]$ and $[\mathbf{4}]$.

Furthermore, the exponent of convergence is also related to the fundamental tone $\lambda_{1}$ of a manifold. When the manifold is compact, it is wellknown that $\lambda_{1}$ is the lowest (first) eigenvalue of the Laplace-Beltrami operator with zero boundary value. The fundamental tone of a manifold is related with its exponent of convergence for complete $n$-dimensional 
Riemannian manifold $M$ with sectional curvatures $K=-1$ :

$$
0 \leq \delta \leq n-1, \quad \lambda_{1}= \begin{cases}(n-1)^{2} / 4, & \text { if } \delta \in[0,(n-1) / 2] \\ \delta(n-1-\delta), & \text { if } \delta \in[(n-1) / 2, n-1]\end{cases}
$$

see $[\mathbf{1 5}$, p. 21] and [19, p. 333]. An extension of this result is proved by Besson, Courtois, and Gallot for manifolds with non-constant negative curvature in [3, Lemma 5.3]: we have $\lambda_{1} \geq \delta(n-1-\delta)$ for every complete $n$-dimensional Riemannian manifold with sectional curvatures $K \leq-1$ (see also [14, Theorem 3.15]). The fundamental tone is also related with isoperimetric inequalities: a general result of Cheeger says that $\lambda_{1} \geq h^{2} / 4$ for every complete Riemannian manifold with infinite volume, where $h$ is the sharp linear isoperimetric constant of the manifold $h=\inf _{A}$ area $(\partial A) / \operatorname{vol}(A)$; it turns out that a lower bound on curvature forces an inequality in the opposite direction (see [5]); hence, to have the linear isoperimetric inequality $(h>0)$ in a manifold with a lower bound on curvature is equivalent to $\lambda_{1}>0$. The linear isoperimetric inequality is closely related to the project of Ancona on the space of positive harmonic functions of Gromov-hyperbolic manifolds (see $[\mathbf{1}],[\mathbf{2}]$ ); in particular, Cao proves that for a large class of Gromov-hyperbolic manifolds (and graphs) the linear isoperimetric inequality implies that the Dirichlet problem at infinity for the LaplaceBeltrami operator is solvable (see [6]). Some of the results of Cao are improved in [13]. Isoperimetric constants are also related with the geometry of ends and large time heat diffusion in Riemannian manifolds (see $[7])$.

Acknowledgments. We would like to thank the referee for her/his valuable comments which have shortened the proof of Theorem 2.1.

\section{Exponent of convergence and Green's function}

An upper bound for the Green's function in a Cartan-Hadamard manifold allows to obtain a criteria for existence of the Green's function in a negatively curved manifold with arbitrary topology:

Theorem 2.1. Let $M$ be a complete $n$-dimensional Riemannian manifold with sectional curvatures $K \leq-1$, and write $M=\tilde{M} / \Gamma$, where $\tilde{M}$ is a universal cover of $M$ and $\Gamma$ is a discrete group of isometries of $\tilde{M}$. If the series

$$
\sum_{\gamma \in \Gamma} \exp \left(-(n-1) d\left(x_{0}, \gamma y_{0}\right)\right)
$$

converges for some $x_{0}, y_{0} \in \tilde{M}$, then there exists the Green's function in $M$. 
Proof: For every $x, y \in \tilde{M}$, triangle inequality gives

$$
\begin{aligned}
\sum_{\gamma \in \Gamma} \exp ( & -(n-1) d(x, \gamma y)) \\
\leq & \sum_{\gamma \in \Gamma} \exp \left(-(n-1)\left(-d\left(x, x_{0}\right)+d\left(x_{0}, \gamma y_{0}\right)-d\left(\gamma y_{0}, \gamma y\right)\right)\right) .
\end{aligned}
$$

Since every $\gamma \in \Gamma$ is an isometry, we have $d\left(\gamma y_{0}, \gamma y\right)=d\left(y_{0}, y\right)$ and

$$
\begin{aligned}
& \sum_{\gamma \in \Gamma} \exp (-(n-1) d(x, \gamma y)) \\
& \quad \leq \exp \left((n-1)\left(d\left(x, x_{0}\right)+d\left(y_{0}, y\right)\right)\right) \sum_{\gamma \in \Gamma} \exp \left(-(n-1) d\left(x_{0}, \gamma y_{0}\right)\right)<\infty .
\end{aligned}
$$

Let us denote by $G_{\tilde{M}}$ the Green's function in $\tilde{M}$. If we denote by $\pi$ the universal cover map $\pi: \tilde{M} \rightarrow M=\tilde{M} / \Gamma$, it is well-known that if the series

$$
\sum_{\gamma \in \Gamma} G_{\tilde{M}}(x, \gamma y)
$$

converges for every $x, y \in \tilde{M}$ with $\pi(x) \neq \pi(y)$, then there exists the Green's function $G_{M}$ in $M$ and furthermore

$$
G_{M}(\pi(x), \pi(y))=\sum_{\gamma \in \Gamma} G_{\tilde{M}}(x, \gamma y) .
$$

The Green function $G_{\mathbb{H}^{n}}(x, y)$ of the $n$-dimensional hyperbolic space $\mathbb{H}^{n}$ is given by $G_{\mathbb{H}^{n}}(x, y)=g\left(d_{\mathbb{H}^{n}}(x, y)\right)$, where $g$ is the following function of the distance $r:=d_{\mathbb{H}^{n}}(x, y)$

$$
g(r)=\frac{1}{\omega_{n}} \int_{r}^{\infty} \frac{1}{(\sinh s)^{n-1}} d s,
$$

where $\omega_{n}$ is the surface area of the unit sphere in $\mathbb{R}^{n}$ (see, e.g., $[\mathbf{1 2}]$ ). Since $\tilde{M}$ is locally isometric to $M$, its sectional curvatures satisfy $K \leq$ -1 , and a standard comparison argument gives that the Green function of $\tilde{M}$ satisfies $G_{\tilde{M}}(x, y) \leq g\left(d_{\tilde{M}}(x, y)\right)$, see [12, Theorem 1].

Let us consider the function

$$
\beta(s):=\left(\frac{e^{s}}{\sinh s}\right)^{n-1}=\left(\frac{2}{1-e^{-2 s}}\right)^{n-1} .
$$

Since $\beta$ is a decreasing function on $(0, \infty)$, we have $\beta(s) \leq \beta\left(r_{0}\right)$ for every $s \geq r_{0}$. Therefore,

$$
\begin{aligned}
g(r) & =\frac{1}{\omega_{n}} \int_{r}^{\infty} \frac{1}{(\sinh s)^{n-1}} d s \\
& \leq \frac{\beta\left(r_{0}\right)}{\omega_{n}} \int_{r}^{\infty} e^{-(n-1) s} d s=\frac{\beta\left(r_{0}\right)}{\omega_{n}(n-1)} e^{-(n-1) r}
\end{aligned}
$$

for every $r \geq r_{0}$. 
Since $\Gamma$ is a discrete group of isometries of $\tilde{M}$, for each fixed choice of $x, y \in \tilde{M}$ with $\pi(x) \neq \pi(y)$, we have that $\Gamma_{x y}:=\{\gamma \in \Gamma: d(x, \gamma y)<1\}$ is a finite set. Hence, (2.1) gives

$$
\begin{aligned}
G_{M}(\pi(x), \pi(y)) & =\sum_{\gamma \in \Gamma_{x y}} G_{\tilde{M}}(x, \gamma y)+\sum_{\gamma \notin \Gamma_{x y}} G_{\tilde{M}}(x, \gamma y) \\
& \leq \sum_{\gamma \in \Gamma_{x y}} G_{\tilde{M}}(x, \gamma y)+\frac{\beta(1)}{\omega_{n}(n-1)} \sum_{\gamma \notin \Gamma_{x y}} e^{-(n-1) d(x, \gamma y)} \\
& \leq \sum_{\gamma \in \Gamma_{x y}} G_{\tilde{M}}(x, \gamma y)+\frac{\beta(1)}{\omega_{n}(n-1)} \sum_{\gamma \in \Gamma} e^{-(n-1) d(x, \gamma y)}<\infty .
\end{aligned}
$$

As a consequence of Theorem 2.1, we deduce our main result.

Theorem 2.2. Let $M$ be a complete $n$-dimensional Riemannian manifold with sectional curvatures satisfying $K \leq-1$ and without Green's function. Then $\delta \geq n-1$.

The inequality in Theorem 2.2 is sharp as the following examples show. As a consequence of (1.1) and Theorem 2.2, if $M$ is any complete $n$-dimensional Riemannian manifold without Green's function and with sectional curvatures $K=-1$, then $\delta=n-1$. Furthermore, there exist manifolds $M$ without Green's function, with sectional curvatures satisfying $K<-1$, and $\delta$ as close as we want to $n-1$ : If $(M, g)$ is any complete $n$-dimensional Riemannian manifold without Green's function and with sectional curvatures satisfying $K=-1$, for each $\varepsilon>0$ let us define the Riemannian metric $g_{\varepsilon}:=(1+\varepsilon)^{-2} g$. It is easy to check that $\left(M, g_{\varepsilon}\right)$ has sectional curvatures $K=-(1+\varepsilon)^{2}<-1$ and $\delta=$ $(1+\varepsilon)(n-1)$.

\section{References}

[1] A. Ancona, Negatively curved manifolds, elliptic operators, and the Martin boundary, Ann. of Math. (2) 125(3) (1987), 495-536. DOI : $10.2307 / 1971409$.

[2] A. Ancona, Positive harmonic functions and hyperbolicity, in: "Potential Theory - Surveys and Problems" (Prague, 1987), Lecture Notes in Math. 1344, Springer, Berlin, 1988, pp. 1-23. DOI: 10.1007/BFb0103341.

[3] G. Besson, G. Courtois, and S. Gallot, Rigidity of amalgamated products in negative curvature, J. Differential Geom. 79(3) (2008), 335-387. 
[4] C. J. Bishop And P. W. Jones, Hausdorff dimension and Kleinian groups, Acta Math. 179(1) (1997), 1-39. DOI: 10.1007/BF02392718.

[5] P. Buser, A note on the isoperimetric constant, Ann. Sci. École Norm. Sup. (4) 15(2) (1982), 213-230.

[6] J. CAO, Cheeger isoperimetric constants of Gromov-hyperbolic spaces with quasi-poles, Commun. Contemp. Math. 2(4) (2000), 511-533. DOI: 10.1142/S0219199700000232.

[7] I. Chavel and E. A. Feldman, Isoperimetric constants, the geometry of ends, and large time heat diffusion in Riemannian manifolds, Proc. London Math. Soc. (3) 62(2) (1991), 427-448. DOI: $10.1112 / \mathrm{plms} / \mathrm{s} 3-62.2 .427$.

[8] J. L. FERnÁndez, On the existence of Green's function in Riemannian manifolds, Proc. Amer. Math. Soc. 96(2) (1986), 284-286. DOI: $10.2307 / 2046168$.

[9] J. L. Fernández And M. V. Melián, Bounded geodesics of Riemann surfaces and hyperbolic manifolds, Trans. Amer. Math. Soc. 347(9) (1995), 3533-3549. DOI: 10.2307/2155022.

[10] J. L. FERnández AND M. V. Melián, Escaping geodesics of Riemannian surfaces, Acta Math. 187(2) (2001), 213-236. DOI : 10.1007 /BF02392617.

[11] J. L. FERnÁndez And J. M. Rodríguez, The exponent of convergence of Riemann surfaces. Bass Riemann surfaces, Ann. Acad. Sci. Fenn. Ser. A I Math. 15(1) (1990), 165-183. DOI: 10.5186/ aasfm.1990.1510.

[12] A. G. Losev, Harmonic functions on manifolds of negative curvature, (Russian), Mat. Zametki 40(6) (1986), 738-742, 829; English translation: Math. Notes 40(6) (1986), 915-917. DOI: 10.1007/BF 01158350.

[13] A. Martínez-PÉrez and J. M. Rodríguez, Cheeger isoperimetric constant of Gromov hyperbolic manifolds and graphs, Preprint (2016). arXiv: 1605.04394.

[14] M. V. Melián, J. M. Rodríguez, And E. Tourís, Escaping geodesics in Riemannian surfaces with variable negative curvature, Submitted (2017).

[15] P. J. Nicholls, "The Ergodic Theory of Discrete Groups", London Mathematical Society Lecture Note Series 143, Cambridge University Press, Cambridge, 1989. DOI : 10.1017/CB09780511600678.

[16] S. J. PAtTerson, Diophantine approximation in Fuchsian groups, Philos. Trans. Roy. Soc. London Ser. A 282(1309) (1976), 527563. DOI : $10.1098 /$ rsta.1976.0063. 
[17] F. Paulin, On the critical exponent of a discrete group of hyperbolic isometries, Differential Geom. Appl. 7(3) (1997), 231-236. DOI: $10.1016 / \mathrm{S} 0926-2245(96) 00051-4$.

[18] L. Sario, M. Nakai, C. Wang, and L. O. Chung, "Classification Theory of Riemannian Manifolds. Harmonic, Quasiharmonic and Biharmonic Functions", Lecture Notes in Mathematics 605, Springer-Verlag, Berlin-New York, 1977. DoI: 10.1007/BFb0064417.

[19] D. Sullivan, Related aspects of positivity in Riemannian geometry, J. Differential Geom. 25(3) (1987), 327-351.

[20] C. YUE, The ergodic theory of discrete isometry groups on manifolds of variable negative curvature, Trans. Amer. Math. Soc. 348(12) (1996), 4965-5005. DOI: 10.1090/S0002-9947-96-01614-5.

María V. Melián and Eva Tourís:

Departamento de Matemáticas

Universidad Autónoma de Madrid

Campus de Cantoblanco

28049 Madrid

Spain

E-mail address: mavi.melian@uam.es

E-mail address: eva.touris@uam.es

José M. Rodríguez:

Departamento de Matemáticas

Escuela Politécnica Superior

Universidad Carlos III de Madrid

Avenida de la Universidad 30

28911 Leganés (Madrid)

Spain

E-mail address: jomaro@math.uc3m.es

Primera versió rebuda el 6 de juliol de 2016, darrera versió rebuda el 2 de desembre de 2016 . 